\title{
Size and Environment Effect on the Room Temperature Plastic Deformation of Ceramic Nanoparticles
}

\author{
I. Issa $^{1}$, L. Joly-Pottuz ${ }^{1}$, J. Amodéo ${ }^{1}$, J. Réthoré ${ }^{2}$, D. J. Dunstan ${ }^{3}$, T. Epicier ${ }^{1}$, I. Jenei ${ }^{4}$, D. Stauffer ${ }^{5}$, \\ C. Esnouf ${ }^{1}$, V. Garnier ${ }^{1}$ and K. Masenelli-Varlot ${ }^{1}$ \\ 1. Univ Lyon, INSA-Lyon, MATEIS, CNRS UMR 5510, F-69621 Villeurbanne, France. \\ 2. Univ Lyon, INSA-Lyon, LaMCoS, CNRS UMR 5259, F-69621 Villeurbanne, France. \\ 3. School of Physics and Astronomy, Queen Mary University of London, London E1 4NS, England. \\ 4. Univ Lyon, Ecole Centrale de Lyon, LTDS, CNRS UMR 5513, F-69134 Ecully, France. \\ ${ }^{5 .}$ Hysitron Inc, 9625 W 76th St., Minneapolis, MN 55344, USA.
}

Materials become more and more nanostructured and nanoparticles are increasingly used in several applications fields. A challenge lies in the in situ microstructural characterization of such materials as it can give access to valuable pieces of information regarding the microstructural changes induced by their use. As far as mechanical properties are concerned, the availability of dedicated TEM (Transmission Electron Microscopy) holders equipped with force and displacement sensors is of a very high interest to test, in situ, the behaviors of such nanometer-sized objects [1].

Many studies are nowadays dedicated to the mechanics of metals at the nano-scale. On ceramic materials, a few works have shown that these materials can exhibit significant plastic deformation, whereas the corresponding bulk materials are brittle [2, 3, 4]. A better comprehension of the mechanisms involved in the deformation of ceramics at the nanoscale could help optimizing their fabrication process.

In the first part, we will present results obtained on initially dislocation-free $\mathrm{MgO}$ nanocubes of sizes ranging between 60 and $450 \mathrm{~nm}$. The contrast in the images (see Figure 1) will be compared with simulation results obtained by Molecular Dynamics. The deformation mechanism is found to involve the nucleation and propagation of $1 / 2<110\rangle\{110\}$ dislocations [4]. Moreover, a mechanical behavior law is identified from the images and the force-displacement curves through Digital Image Correlation and Finite Elements Simulations [5]. A strong effect of the nanocube size on the yield stress can be observed. This effect is investigated using maximum likelihood statistical methods and the parameters of the scaling law are compared with literature data on other types of materials.

In a second part, we will present initial experiments on ceria nanocubes tested in a controlled environment (i.e. under gas pressure). Indeed, ceria undergoes crystallographic changes under high vacuum. Introducing oxygen inside the chamber limits or avoids the reduction of $\mathrm{CeO}_{2}$ nanocubes induced more or less rapidly by the electron beam. Compression cycles on the same nanocube, tested either under high vacuum or under oxygen (see Figure 2), strongly suggests that ceria reduced to $\mathrm{Ce}_{2} \mathrm{O}_{3}$ under the effect of an intense electron flux has a smaller Young modulus than unreduced or fully oxidized ceria. The formation of dislocations and stacking faults is discussed from atomic resolution images acquired during compression [6].

\section{References:}

[1] Q Yu, M Legros and AM Minor, MRS Bulletin 40 (2015), p. 62. 
[2] S Korte et al, Acta Mater 59 (2011), p. 7241.

[3] E Calvié et al, J Eur Ceram Soc 32 (2012), p. 2067.

[4] I Issa et al, Acta Mater 86 (2015), p. 295.

[5] E Calvié et al, Mater Lett 119 (2014), p. 107.

[6] This work is partly performed within the framework of the LABEX iMUST (ANR-10-LABX-0064) of Université de Lyon, within the program "Investissements d'Avenir" (ANR-11-IDEX-0007) operated by the French National Research Agency (ANR). The authors thank the CLYM (Consortium Lyon-Saint Etienne de Microscopie, www.clym.fr) for the access to the microscopes and A.K.P. Mann, Z. Wu and S.H. Overbury (ORNL, USA) for having provided the ceria samples.

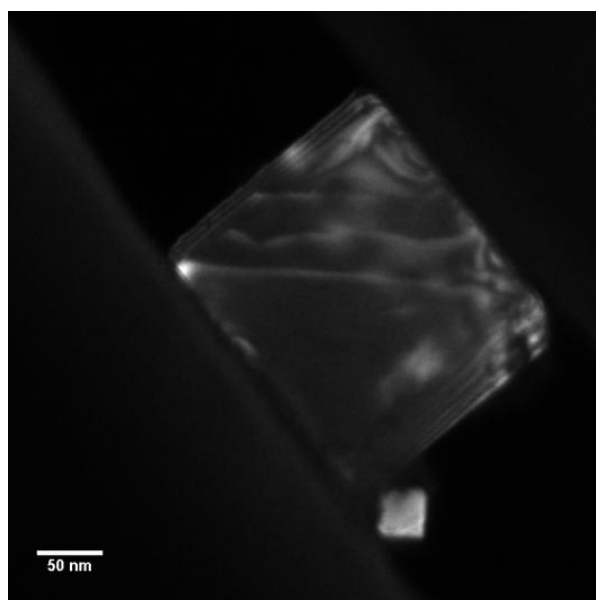

Figure 1. Weak-Beam Dark Field Image of a $195 \mathrm{~nm}$ nanocube during compression ( $\mathrm{g}=[002])$. The straight lines correspond to screw $1 / 2$ [011] (01-1) dislocations propagating in the nanocube.
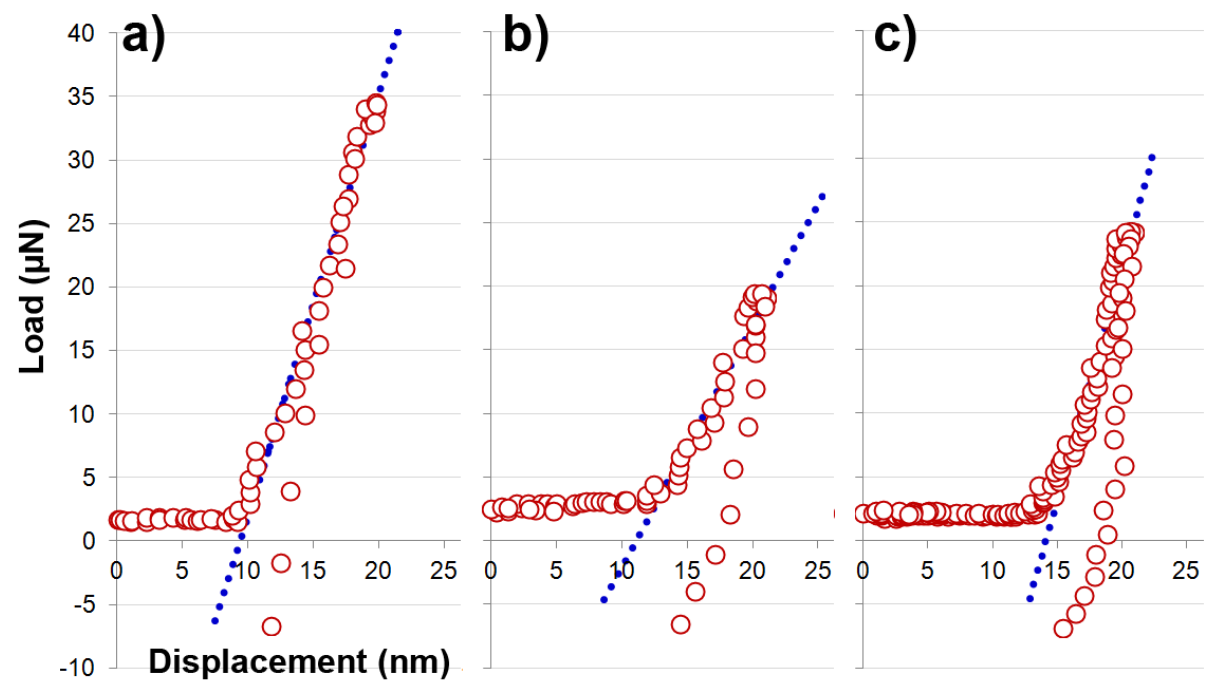

Figure 2. Comparison of slopes in the pseudo-elastic region of stress-strain curves recorded successively on the same nanocube in high vacuum: a) un-reduced $\mathrm{CeO}_{2}$ (low electron dose), b) reduced $\mathrm{Ce}_{2} \mathrm{O}_{3}$ (high electron dose) and re-oxidized ceria under air at 2.6 mbar. 